\title{
Rapid Thermal Oxidation of Ge-rich Strained Layers
}

\author{
S. Das, S. Chakraborty, S. Bhattacharya, M. Bain, J. McCarthy, \\ B. M. Armstrong, H. S. Gamble, G. K. Dalapati, S. K. Samanta, T. S. Perova, \\ R. A. Moore, and C. K. Maiti
}

\begin{abstract}
In this paper, we report for the first time the electrical properties of ultrathin oxides grown using rapid thermal oxidation (RTO) on strained Ge-rich layers on relaxed-SiGe buffers. Rapid thermal oxidation on strained Ge-rich layer is employed to prevent strain relaxation. Electrical properties of MOS capacitors fabricated using RTO grown oxides directly on strained Ge-rich has been studied in detail using capacitance-voltage (C-V), conductance-voltage (G-V) and current-voltage (I-V) characteristics. Interface trap density, fixed oxide charge density, the frequency dispersion and hysteresis effects of the oxide have been determined. From the I-V characteristics, the current conduction mechanism has also been studied. RTO grown oxides show good electrical properties and may find applications in the future generation Ge-CMOS as a gate dielectric.
\end{abstract}

\section{INTRODUCTION}

As bulk-Si reaches its fundamental limits with the demand of high performance CMOS technologies and faster scaling of the devices, new device structures are implemented to suffice the need [1]. One of the promising candidates is Ge owing to its narrow band gap, high hole mobility (roughly four times that of bulk-Si) and high solubility limits of p-type dopants. With the advent of SiGe technology and its subsequent development with higher $\mathrm{Ge}$ concentration, there has been an urgent need to establish an effective passivation layer on Ge surfaces. In the last few decades, efforts have been made to realize stable oxide layers on $\mathrm{Ge}$ [2]-[4] by various oxidation processes. Unlike $\mathrm{Si}$, however, the lack of a stable native oxide hinders the passivation of $\mathrm{Ge}$ surfaces. Again a number of dielectric like $\mathrm{Ge}$ oxynitride [5], $\mathrm{Ge}_{3} \mathrm{~N}_{4}[6]$ and $\mathrm{Al}_{2} \mathrm{O}_{3}$ [7] have been

S. Das, S. Chakraborty, S. K. Samanta, G. K. Dalapati, and C. K. Maiti are with the Department of Electronics \& ECE, Indian Institute of Technology, Kharagpur 721302, India, E-mail: ckm@ece.iitkgp.ernet.in

S. Bhattacharya, M. Bain, B. M. Armstrong and H. S. Gamble are with the School of Electrical \& Electronic Engineering, The Queen's University of Belfast, United Kingdom, E-mail: sekhar@qub.ac.uk

J. McCarthy, T. S. Perova, and R. A. Moore are with the Department of Electronic \& Electrical Engineering, Trinity College, University of Dublin, Dublin 2, Ireland, E-mail: perovat@tcd.ie attempted on Ge surfaces. However, none of them has been able to offer an equivalent oxide thickness (EOT) of less than $10 \AA$ to advance the current technology beyond sub-20 $\mathrm{nm}$ regime. In this study, we have successfully employed rapid thermal oxidation process for the growth of good quality oxide on strained $\mathrm{Ge}$-rich layers grown on virlual substrates. In case of strained-Ge layers, it is important that the oxidation should not cause strain relaxation. Studies have revealed RTO as a means to prevent strain relaxation due to its low thermal budget [8]. Another advantages of this process are its high temperature ramp up rate (and hence a reduced thermal exposure) and a good control of oxide thickness. Strain relaxation has also been minimized with the use of virtual substrate [9] as growth of SiGe buffer layer on the bulk-Si reduces the dislocation and defects.

\section{EXPERIMENTAL}

The starting substrate was n-type Si (100) with resistivity $10-20 \Omega-\mathrm{cm}$. The wafer was cleaned in four separate baths before loading in the process chamber. First the wafer was dipped in 10:1 HF solution for $1 \mathrm{~min}$ to remove the native oxide. Then they were cleaned in $1: 1$ $\mathrm{H}_{2} \mathrm{SO}_{4}$ and $\mathrm{H}_{2} \mathrm{O}_{2}$ solution, followed by a dip in $10: 1 \mathrm{HF}$ solution for $1 \mathrm{~min}$ to remove the thin oxide formed during cleaning. In the next step, the wafer were cleaned in megasonically agitated solution of $\mathrm{NH}_{4} \mathrm{OH}, \mathrm{H}_{2} \mathrm{O}_{2}$, and $\mathrm{H}_{2} \mathrm{O}$ to remove particles residing on the surface of the wafer and dipped in 10:1 HF solution for $1 \mathrm{~min}$ to remove the oxide. Finally, the wafers were exposed to $48 \% \mathrm{HF}$ for $1 \mathrm{~min}$ for hydrogen surface passivation immediately before loading into load lock. From loadlock the wafer are transferred to the process chamber by robotic arm.

Relaxed graded- $\mathrm{Si}_{1-x} \mathrm{Ge}_{\mathrm{x}}$ buffers with $\mathrm{x}=0-0.6$ of 0.8 $1.0 \mu \mathrm{m}$ thickness were grown by UHV compatible LPCVD at $800^{\circ} \mathrm{C}$. In order to avoid high surface roughness generally relaxed graded buffers are chemically mechanically polished (CMP) to remove the surface roughness associated with mismatched heteroepitaxial layers [10]. This process minimizes subsequent dislocations and reduces defect densities. On this substrate, strained Ge-rich layers of 0.15-0.2 $\mu \mathrm{m}$ thicknesses werc grown in the process chamber and on top of it sacrificial $\mathrm{Si}$ cap layer was deposited. The process gases used were $\mathrm{SiH}_{4}$ and $\mathrm{GeH}_{4}$ and working pressure was kept between 0.092- 
0.150 Torr. The total film thickness was kept between 1$1.2 \mu \mathrm{m}$. The structure of the layers is shown in the Fig. 1 .

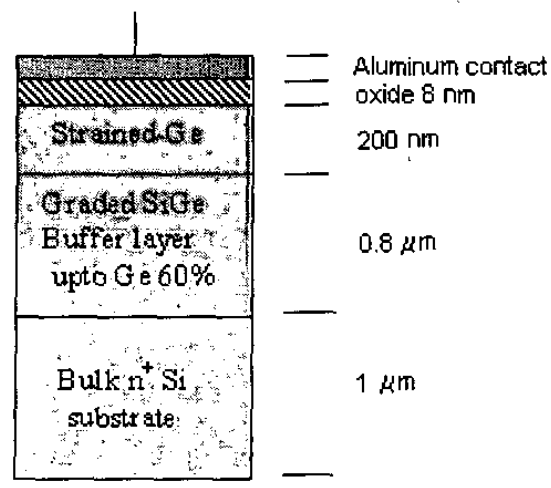

Fig. 1. Schematic diagram of MOS structure.

The oxide was grown on the strained Ge-rich layers by the process of rapid thermal oxidation (RTO) at a temperature of $620^{\circ} \mathrm{C}$ for $105 \mathrm{~s}$. The ramp up rate of the rapid thermal processor is $30^{\circ} \mathrm{Cs}^{-1}$ while the ramp down rate is low. A cross sectional image of strained-Ge structure were obtained from TEM micrograph using PHILLIPS TECHNAI F20. The field emission gun produces a potential of $200 \mathrm{kV}$. Prior to TEM, the sample is subjected to mechanical grinding followed by $\mathrm{Ga}^{+}$ion milling. Raman spectral analysis was registered in backscattering geometry using a RENISHAW 1000 microRaman system equipped with LEICA microscope. The power density was kept below $105 \mathrm{~mW} \mathrm{~cm}^{-2}$ to reduce the sample preheating. The measurements were performed at room temperature with $\mathrm{Ar}^{+}$laser. An 1800 lines $/ \mathrm{mm}$ grating was used in all measurements with spectral resolution of $\sim 2.5 \mathrm{~cm}^{-1}$ per pixels. The 100 times magnifying objectives of the Leica microscope focuses the beam into the spot of about $1 \mu \mathrm{m}$ in diameter. MOS capacitors were then fabricated by depositing aluminum circular dots on the sample of area $1.96 \times 10^{-3} \mathrm{~cm}^{2}$. The electrical measurement including $\mathrm{C}-\mathrm{V}$ dispersion, $\mathrm{I}-\mathrm{V}$ and hysteresis in the $\mathrm{C}-\mathrm{V}$ characteristics were carried out using HP-4061A semiconductor test system.

\section{RESUltS AND DISCUSSIONS}

The cross-sectional TEM image in Fig. 2 shows the complete picture of heterostructure layers. It also reveals an undulation in the film surface as the growth is carried out at an elevated temperature. It is found that the compressively strained-layers starts to undulate at wavelengths approximately $100 \mathrm{~nm}$. It is noticed that the entire Si cap layer have been used up for the growth oxide layers. Fig. 3 shows that the Raman peaks are present at wave numbers of $400 \mathrm{~cm}^{-1}$ and $465 \mathrm{~cm}^{-1}$ due to, Si-Ge and $\mathrm{Si}-\mathrm{Si}$ bonds respectively.

The spectral lines are fitted with Lorentzian or Gaussian functions. The strain level determines the phonon energy obtained in the Raman peaks and thus can be used to extract strain of the heterolayers. The strain in the layer was found to be 0.01688 . Similar peaks for the $\mathrm{Si}_{0.3} \mathrm{Ge}_{0.7}$ are shown in [11] and [12].

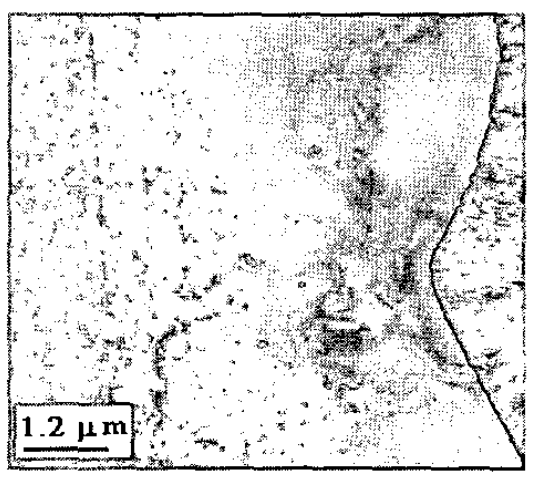

Fig. 2. TEM micrograph of strained-Ge layers.

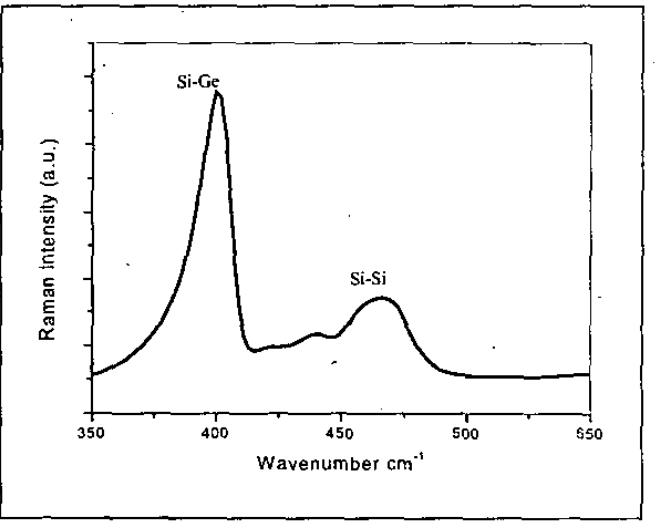

Fig. 3. Raman spectra of strained-Ge.

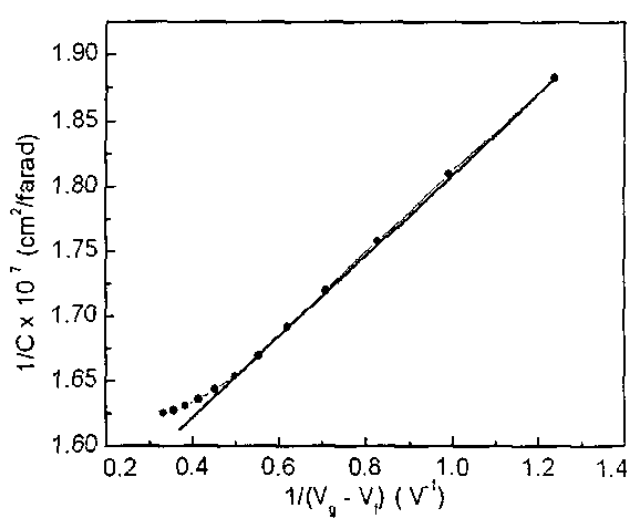

Fig. $4.1 / \mathrm{C}$ vs. $1 /\left(\mathrm{V}_{\mathrm{g}}-\mathrm{V}_{\mathrm{f}}\right)$ plot. 
The thickness is measured from $1 / \mathrm{C}$ vs. $1 /\left(\mathrm{V}_{\mathrm{g}}-\mathrm{V}_{\mathrm{fb}}\right)[13]$ plot at high frequency found to be $80 \pm 3 \AA$ as shown in Fig. 4. The C-V dispersion (Fig. 5) shows a shift in the flatband voltage in the positive bias and stretch of $\mathrm{C}-\mathrm{V}$ characteristics with the increase of frequency. This may be attributed to the presence of interface traps and mobile oxide charges. The interface trap density is evaluated by Hill's method at the midgap energy value from $\mathrm{C}-\mathrm{V}$ and $\mathrm{G}-\mathrm{V}$ characteristics is found to be $7.5 \times 10^{11} \mathrm{~cm}^{-2} \mathrm{ev}^{-1}$ and the fixed oxide charge density obtained from $\mathrm{C}-\mathrm{V}$ characteristics is found to be $1.35 \times 10^{12} \mathrm{~cm}^{-2}$. Similar results were reported by Benamura et al. [14]. It may be noticed that at a comparatively low frequency the capacitance in the accumulation decreases with the increase of applied field which may be due to conduction of charges across the oxide layers. The dispersion in the inversion is mainly attributed to the effect of resistance of at the semiconductor oxide interface and the substrate back contact. The hysteresis in the high frequency $\mathrm{C}-\mathrm{V}$ characteristics is obtained from double voltage sweep from inversion to accumulation and back to inversion. The hysteresis voltage is found to be $56 \mathrm{mV}$ (Fig. 6). Similar results were also reported by Chui et al. [15].

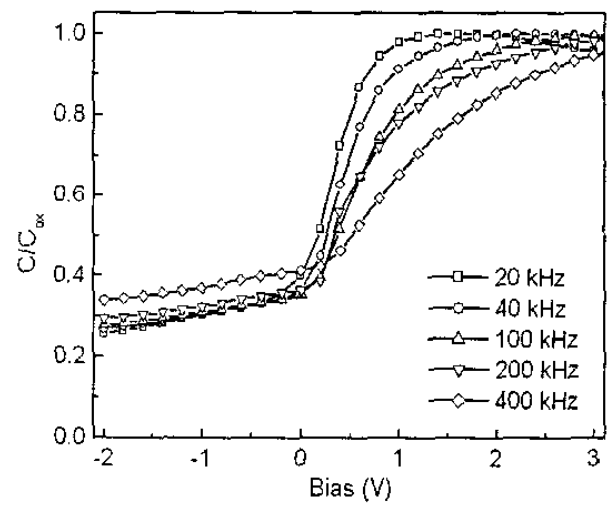

Fig. 5. C-V dispersion of RTO grown MOS on strained-Ge.

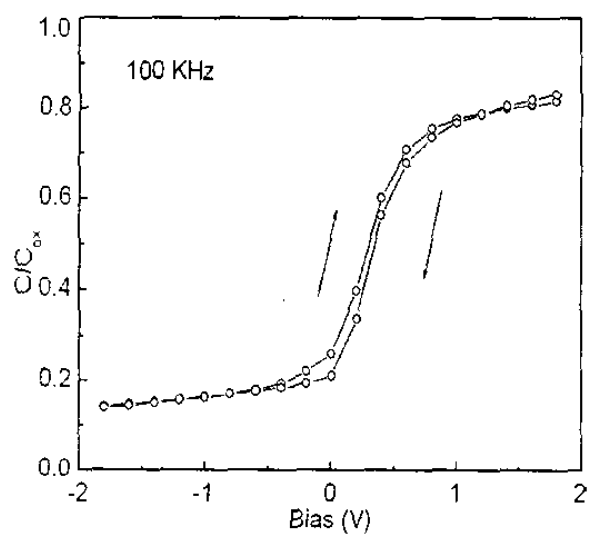

Fig. 6. C-V characteristics of RTO grown MOS.

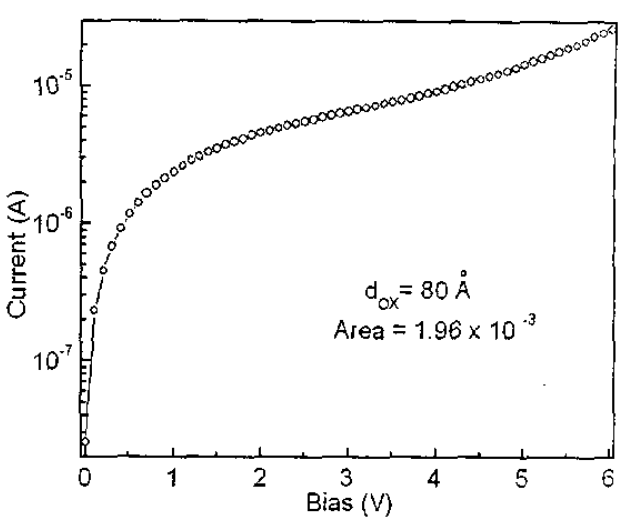

Fig. 7. Typical I-V characteristics of RTO grown MOS.

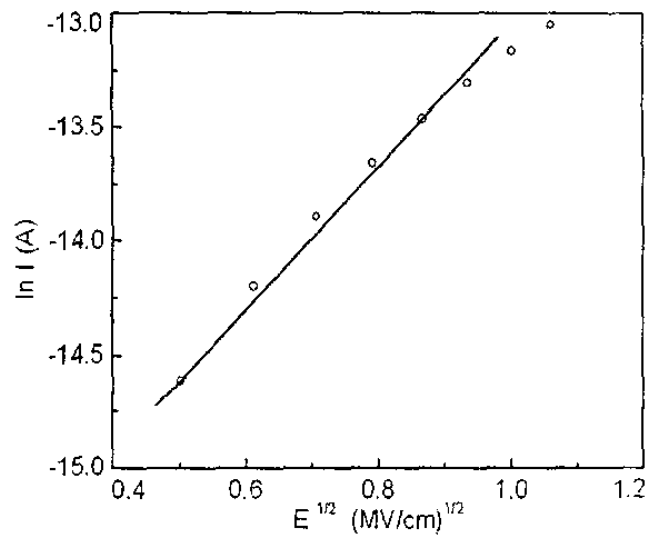

Fig. 8. In (I) vs $E^{1 / 2}$ plot: Schottky emission characteristics.

The $1-V$ characteristics in Fig. 7 show the current in the accumulation region for n-type substrate. To determine the dominant leakage current mechanism in the oxide layer a plot of $\ln (\mathrm{I})$ versus $E^{1 / 2}$ (Schottky emission mechanism) is commonly used (Fig. 8). RTO grown oxide on strained-Ge shows clearly a linear relationship and the conduction mechanism is dominated by the Schottky emission at room temperature [16]. It is noted that the Schottky emission occurs as a result of lowering of the columbic potential barrier due to free carrier generation out of the defect centers and traps under the applied electric field. The phenomena may be considered as electrode limited, occurring at low voltage where carriers at the metal surface or semiconductors transit above potential barrier and are prominent in thin films.

\section{CONCLUSION}

The results of our studies on the electrical characteristics of MOS capacitors fabricated using RTO 
grown oxides directly on strained Ge-rich layers reveal a reasonably good reliability. The leakage current through the oxide is comparable to that of conventional $\mathrm{SiO}_{2}$ grown on Si-substrates. The low hysteresis voltage also indicates a good reliability of the oxides grown directly on strained Ge-rich layers. It is expected that the strained Ge-rich heterolayers can possibly immerge as a suitable candidate for the next generation high performance Ge-CMOS technology.

\section{REFERENCES}

[1] The International Technology Roadmap for Semiconductor, Semiconductor Industry Association, 2001.

[2] V. Craciun, 1. W. Boyd, B. Hutton, and D. Williams, "Characteristics of dielectric layers grown on Ge by low temperature vacuum ultraviolet-assisted oxidation", Appl. Phys. Lett., vol. 75, pp.1261-1263, 1999.

[3] T. Lange, W. Njoroge, H. Weis, M. Backers, and M. Wuttig, "Physical properties of thin $\mathrm{GeO}_{2}$ produced by reactive DC magnetron sputtering", Thin Solid Films, vol. 365, pp. 82-89, 2000.

[4] Y. Wang, Y. Z. Hu, and E. A. Irene, "Electron cyclotron resonance plasma and thermal oxidation mechanisms of germanium", J. Vac. Sci. Technol. A, vol. 129, pp. $1309-$ 1314, 1994.

[5] D. J. Hymes and J. J. Rosenberg, "Growth and material characterization of native germanium oxynitride thin film on germanium", J. Electrochem. Soc., vol. 135, pp. 961-965, 1988.

[6] T. Yashiro, "Determination of trap levels in $\mathrm{Ge}_{3} \mathrm{~N}_{4}$ and barrier energies at $\mathrm{Ge}_{3} \mathrm{~N}_{4}-\mathrm{Ge}$ interface by $\mathrm{C}-\mathrm{V}$ characteristics", Jpn. J. Appl. Phys., vol. 10, pp. 1691-1697, 1971.
[7] B. G. Segda, M. Jacquet, C. Caapera, G. Baud, and J. P. Besse, "Study and optimization of alumina and germanium dioxide and their multilayer capacitance", Nucl. Instr. Meth. Phys. Res. B, vol. 170, pp. 105-114, 2000.

[8] S. K. Samanta, S. Chatterjee, W. K. Choi, L. K. Bera, H. D. Banerjee, and C. K. Maiti, "Reliability of ultrathin $(<2 \mathrm{~nm})$ oxides on strained SiGe heterolayers", Semicond. Sci Technol, vol. 18, pp. 33-38, 2003.

[9] E. Kasper, K. Lyutovich, M. Bauer, and M. Oehme, "New virtual substrate concept for vertical MOS transistors", Thin Solid Films, vol. 336, pp. 319-322, 1998.

[10] M. T. Currie, S. B. Samavedam, T. A Langdo, C. W. Leitz, and E.A. Fitzgerald, "Controlling threading dislocation densities in $\mathrm{Ge}$ on $\mathrm{Si}$ using graded $\mathrm{SiGe}$ layers and chemicalmechanical polishing", Appl. Phys. Lett., vol. 72, pp. 17181720,1998

[11] M. I. Alonso and K. Winer, "Raman spectra of $\mathrm{c}-\mathrm{Si}_{1-x} \mathrm{Ge}_{x}$ alloys", Phys. Rev., vol. 39, pp. 10056-10062, 1989.

[12] H. Yin, R. L. Peterson, K. D. Hobart, S. R. Shieh, T. S. Duffy, and J. C. Sturm, "Relaxed SiGe Layers with High Ge Content by Compliant Substrate", Mat. Res. Soc., vol. 768 , 2003.

[13] G. Ghiboudo, S. Bruyere, T.Devoivre, B. DeSalvo, and E. Vincent, "Improved method for the oxide thickness extraction in MOS structure with Ultrathin Gate Dielectrics", IEEE Trans. Semicond. Manufact., vol. 13, pp. 151-158, 2000.

[14] Z. Benamara, S. Tizzi, M. Chellai, and B. Gruzza, "Experiments in MIS structure based on germanium and improvements of the interfacial properties", Materials Chem and Phys., vol. 62, pp. 273-276, 2000

[15] C. O. Chui, S. Ramanathan, B. B. Triplett, P. C. McIntrye, and K.C. Saraswat, "Germanium MOS capacitors Incorporating Ultrathin High-K Gate Dielectric", IEEE Electron Dev. Lett., vol. 23, pp. 473-475, 2002

[16] J. P. Chang and Y. S. Lin, "Dielectric property and conduction mechanism of ultrathin zirconium oxide films", Appl. Phys. Lett., vol. 79, pp. 3666-3669, 2001. 